\title{
Dioxinas e Dibenzofuranos no meio Ambiente
}

\author{
J.C.M.B ORDADO*, H.M.S.FERREIRA**, J.F.P. GOMES***
}

\section{INTRODUÇÃO}

Ao longo deste século verificou-se um grande desenvolvimento da indústria química, nomeadamente na produção industrial de compostos de síntese, tendo-se que uma das grandes áreas de desenvolvimento ocorreu ao nível da química dos compostos organoclorados. O cloro, apesar dos problemas de segurança e toxicidade que sempre apresentou, foi um elemento que permitiu o aparecimento de diversos polímeros - dos quais o mais vulgarizado é o policloreto de vinilo (PVC) com inúmeras aplicações dos herbicidas e pesticidas como o DDT e fluidos dieléctricos e aerossóis designados genericamente por clorofluoro-carbonados (CFCs). O fabrico intenso de compostos organoclorados que se verificou a partir da década de 40 , aliado à sua grande estabilidade biológica e molecular, tem vindo, progressivamente, a colocar problemas ambientais uma vez que os compostos deste tipo, por regra bastante tóxicos, se têm vindo a acumular na natureza de forma preocupante. Além disso, o esforço humano em reduzir a acumulação dos compostos acima referidos conduz, por vezes, à produção de compostos de toxicidade ainda mais elevada como são as dibenzo-para-dioxinas policloradas, correntemente designadas por dioxinas (PCDDs - "PolyChlorinated DibenzoDioxins"). Algumas dioxinas e dibenzofuranos são compostos cancerígenos para os animais e também para os seres humanos. Embora ainda não sejam totalmente conhecidos todos os efeitos das dioxinas nem estejam explicados todos os mecanismos pelos quais elas actuam, já se encontram definitivamente estabelecidos alguns aspectos toxicológicos que permitem classificá-las como das substâncias mais tóxicas conhecidas.

No que diz respeito à sua origem, tem-se que estes compostos nunca foram produzidos industrialmente com um objectivo concreto mas resultam, como produtos indesejáveis, de reacções secundárias em diversos processos industriais das indústrias química, do papel e da celulose, me- talúrgicas (e particularmente, siderúrgicas), de desengorduramento de metais e de fabrico de materiais poliméri$\cos$.

Contudo, uma outra fonte particularmente importante destes compostos reside nos processos de combustão que ocorrem em incineradores, quer se trate de incineradores de resíduos urbanos, hospitalares ou industriais, quer de plásticos e de lamas de depuração de tratamento de águas e efluentes líquidos, particularmente no caso em que os teores em compostos clorados sejam significativos. Dada a elevada perigosidade dos compostos anteriormente referidos, tem-se vindo a procurar diminuir os níveis de emissão e os respectivos impactes ambientais daí decorrentes. Esta diminuição passa pela introdução de novos processos, incluindo a optimização da combustão nos sistemas de incineração, mas também pelo uso de combustíveis isentos de cloro e pela substituição de produtos e matérias-primas industriais contendo cloro. Concomitantemente, têm vindo a ser desenvolvidas técnicas cada vez mais precisas de doseamento analítico destas substâncias, embora seja este um campo onde se verifica ainda a necessidade da realização de trabalhos de Desenvolvimento de métodos, por forma a permitir uma correcta monitorização ambiental que, de momento, ainda é difícil de efectuar para alguns tipos de matrizes, em particular em amostras de natureza biológica.

As dioxinas passaram a ser "tristemente célebres" a partir de acidentes graves de derrames em unidades industriais do sector químico, como foram o de Times Beach, Missouri, EUA, no início dos anos 70, e principalmente o de Seveso, Milão, Itália, que ocorreu em 1976. No dia 10 de Julho de 1976 verificou-se uma libertação extemporânea de uma mistura de compostos químicos contendo 2,3,7,8-tetraclorodibenzo-p-dioxina (TCDD), para a atmosfera, a partir de uma fábrica da indústria química situada na localidade de Seveso, atingindo uma área considerável, densamente povoada, nas proximidades de Milão. A ocorrência deste acidente veio mostrar a necessidade da Indústria conhecer em detalhe os riscos e perigosidade dos produtos libertados em condições de emergência ou acidente, mas também as dificuldades, tanto da indústria como das autoridades, em fazer face a emergências desta natureza. Eata circunstância levou ao aparecimento de uma nova filosofia de notificação e prevenção de acidentes industriais ditos "graves", consubstanciada por legislação europeia especílica conhecida como a Directiva Comunitária Seveso. Além disso ficaram ainda evidentes os poucos conhecimentos de que se dipunha acerca das dioxinas, em termos da sua química, mecanismos de formação e de reacção, e toxicidade.

\section{CARACTERÍSTICAS FÍSICAS E QUÍMICAS DAS DIOXINAS E DIBENZOFURANOS}

A primeira dioxina clorada foi sintetizada em 1872 por Merz e Weith, mas a estrutura do composto só ficou completamente esclarecida em 1957 [1]. A primeira síntese de TCDD também foi efectuada em 1957 [2], tendo-se verificado que, em ambos os casos, os técnicos de laboratório envolvidos nos trabalhos foram hospitalizados com sintomas de intoxicação [3]. Entre esta data e o acidente de Seveso, encontram-se documentados diversos acidentes ocorridos no processo de produção de 2,4,5-triclorofenol a partir de 1,2,4,5-tetraclorobenzeno, que atingiram mais de 410 trabalhadores. No entanto, a toxicidade da TCDD só passou a ser devidamente estudada a partir do acidente de Seveso [4]. Os derivados clorados das dibenzo-para-dioxinas são compostos orgânicos com analogias estruturais com os hidrocarbonetos aromáticos policíclicos (PAHs). Os átomos de hidrogénio das posições 1 a 8 podem ser todos substituídos por átomos de cloro, havendo assim um total de 75 possibilidades de ocorrência de derivados clorados diferentes, de entre os quais a 2,3,7,8-tetraclorodibenzo-p-dioxina $(2,3,7,8$-PCDD ou TCDD), é o que apresenta o maior 
grau de toxicidade [5 e 6]. A investigação sistemática destes compostos efectuada a partir de 1976 revelou aspectos da elevada toxicidade das dioxinas e, em particular, que a TCDD é declaradamente a substancia cancerígena mais potente, em termos de organismos humanos, alguma vez avaliada pela USEPA, a Agência de Protecção Ambiental Americana [6]. Isto significa que mesmo a exposição a quantidades extraordinariamente diminutas deste composto pode provocar efeitos, na saúde, muito sérios e irreversíveis. Diversos estudos toxicológicos em roedores, peixes e macacos têm vindo a demonstrar que a exposição a TCDD tem efeitos carcinogéni$\cos$, reduz as defesas imunológicas e a fertilidade [5].

Apesar da quase total inexistência de estudos em humanos, por razões compreensíveis, reconhece-se que a persistência de TCDD no organismo é elevada, sendo estimada a sua meiavida no organismo em cerca de 7 anos. Até agora, os efeitos observáveis em indivíduos expostos a dioxinas têm estado associados a sindromas não letais como o aparecimento de cloroacne, a activação de alguns enzimas (tais como os da família dos citocromos $\mathrm{P} 450$ ) e a possível redução da contagem de espermatozóides, bem como alterações do sistema imunológico e da velocidade de coagulação do sangue [6]. A exposição dos seres humanos a altos níveis de concentração de dioxinas ocorrida durante os graves acidentes industriais anteriormente referidos resultou, em primeiro lugar em afecções tais como cloroacne e doenças do fígado [7]. Estudos epidemiológicos efectuados sobre as populações expostas e publicados depois desta data [8], estabelecem definitivamente que as dioxinas são promotores/carcinogénicos como já havia sido demonstrado em animais. Os indícios existentes relativos à elevada toxicidade são mais do que suficientes para impor a identificação das fontes de dioxinas e reduzir as emissões para o ambiente, evitando assim contaminações através da cadeia alimentar que é uma das principais fontes próximas de contaminação do homem.

\section{FONTES PRINCIPAIS EMISSORAS DE DIOXINAS E DIBENZOFURANOS PARA A ATMOSFERA}

Historicamente, as fontes emissoras de dioxinas e dibenzofuranos classificam-se em três categorias principais:

\section{a) Industriais :}

- processos da indústria química

- processos da indústria de celulose e papel

- processos metalúrgicos e siderúrgicos

- desengorduramento de metais

- Labrico de retardantes de chama contendo bromo e/ou cloro

b) Processos de combustão

b.1) Fontes estacionárias :

- incineradoras de resíduos municipais

- incineradoras de resíduos tóxicos e perigosos

- incineradoras de resíduos hospitalares

- combustão de lamas de depuração de ETARs metais

- processos de reciclagem de

b.2) fontes móveis :

- exaustão de gases de veículos automóveis

- incêndios florestais e incêndios controlados em actividades agrícolas

- fumo de cigarros

b.3) fontes acidentais:

- combustão de policlorobifenilos (PCB), PVC, incêndios envolvendo outros materiais plásticos, etc.

c) Fontes secundárias:

- exaustão de gases provenientes de aterros e áreas contaminadas

- aplicações decorrentes da utilização de lamas de depuração de Estaçōes de Tratamento de Águas Residuais (ETAR)

\subsection{Indústria química e indús- trias paraquímicas}

No que diz respeito aos processos de produção da indústria química, são de salientar os seguintes processos como potenciais geradores de dioxinas e dibenzofuranos como produtos secundários do processo de fa- brico por ordem decrescente de importância :

- produção de clorofenóis e seus derivados

- produção de clorobenzeno e clorobenzenos substituídos

- síntese de compostos clorados alifáticos

- processos que recorrem a intermediários clorados

- produção de compostos clorados inorgânicos

- processos que utilizam solventes e catalisadores clorados

- manufactura de retardantes de chama contendo bromo e/ou cloro

$\mathrm{A}$ indústria química $\mathrm{e}$ as industrias paraquímicas que utilizam compostos de cloro têm vindo a aceitar a necessidade de contribuir para o estudo e resolução do problema, investindo em tecnologias mais limpas ou, quando possível, isentas de cloro. O melhor exemplo a este respeito é a evolução recente que se verificou no sector de produção de pasta de celulose, em que foram inicialmente desenvolvidas as tecnologias ECF ("Elemental Chlorine Free" - isenta de cloro elementar) e posteriormente TCF ("Total Chlorine Free", isenta de quaisquer compostos de cloro). No caso deste sector industrial a matéria-prima, a madeira, é a fonte de compostos orgânicos que sofrem halogenação pelo contacto com os agentes clorados que intervêm no processo de branqueamento, tendo a formação de compostos organoclorados vindo a ser reconhecida nesses processos de fabrico [9]. Rosenberg et al [10] efectuou um estudo de caracterização das atmosferas ocupacionais da unidade de branqueamento e da máquina de papel de uma fábrica de produção de pasta na Finlândia. Foram encontradas concentrações (expressas como I-TEQ), no ar, de $2,3,7,8$ dioxinas e dibenzofuranos que variavam entre 0,04 e $1,9 \mathrm{pg} / \mathrm{m}^{3}$, tendo-se que os dibenzofuranos se encontravam presentes em quase todas as amostras e as dioxinas em muito poucas. É de registar que se verificou, neste estudo, que as concentrações mais elevadas de ambos os compostos foram obtidas nas amostras colhidas na unidade de branqueamento quan- 
do esta processava madeiras de bétula. Os compostos halogenados como as dioxinas e dibenzofuranos que se formam no processo de produção de pasta de celulose são emitidos para o ambiente exterior nos efluentes gasosos e em suspensão nos efluentes líquidos, em particular no caso dos provenientes das unidades de branqueamento de pasta. Uma vez que se trata de compostos muito estáveis, extraordinariamente difíceis de degradar (são estáveis a temperaturas superiores a 700 C, o que é uma razão para a sua elevada persistência), estas espécies ficam em grande parte retidas nas lamas quando se efectua o tratamento de efluentes líquidos. Essas lamas podem sofrer vários tipos de utilização, entre os quais a reciclagem para produção de papel se o teor e qualidade de fibras ainda for apreciável, embora muitas vezes sejam depositadas em aterro ou incineradas em caldeiras de biomassa. Se tal acontece, as dioxinas presentes nas lamas podem vir a ser, em parte, emitidas para a atmosfera, dada a dificuldade de destruir esses compostos, mesmo às altas temperaturas verificadas nas unidades de combustão [11]. Além disso, verificase a ocorrência destes compostos nas cinzas resultantes da operação dessas caldeiras, de acordo com os estudos de Someshwar et al. [12]. Estes autores, através de uma série de campanhas de medição efectuadas em unidades nos Estados Unidos, encontraram um teor de emissão médio, para estas caldeiras, de $4,910^{-10} \mathrm{~kg}$ I-TEQ por tonelada de lamas alimentada. Em incineradores de resíduos sólidos urbanos a emissão típica média era em 1987 de 1,4 x 10-7 kg I-TEQ por tonelada de resíduos. Nos modernos incineradores de resíduos sólidos as emissões típicas são hoje mais de 10000 vezes inferiores.

\subsection{Indústria Siderúrgica}

Outro sector industrial em que se verifica ocorrerem emissões não neglicenciáveis de dioxinas é o sector siderúrgico. De uma pesquisa efectuada, recentemente, sobre o sector siderúrgico sueco chegou-se ao quantitativo global de $0,8 \mathrm{ng}$ I-TEQ/g de partículas emitidas [13], considerando a Agencia de Protecção Ambiental Sueca que este sector é a principal fonte de dioxinas neste pais. Também na Suécia e na Holanda foram detectados, em unidades típicas de sinterização, teores nos efluentes gasosos superiores a $3 \mathrm{ng} / \mathrm{Nm}^{3}+$ o que faz corresponder (nesses países) a um valor médio de cerca de $24 \mathrm{~g} \mathrm{I}$ -TEQ/ano por unidade fabril que integre uma unidade desse tipo. Do mesmo modo, em partículas emitidas a partir de processos de fundição, tem vindo a ser detectada a presença de dioxinas em quantidades superiores a $22.7 \mathrm{ng} I-T E Q / g$. Se bem que existam poucos estudos a respeito deste sector para a Europa do Sul, é de prever que os teores sejam também elevados nesta região, tanto mais que aqui se verifica, geralmente, a existência de unidades antigas, de pequenas dimensões, e que processam grandes quantidades de sucata de metais ferrosos, muitas vezes contaminada com outros materiais.

\subsection{Incineração de resíduos}

Por último, o sector da incineração de resíduos, quer se trate de resíduos sólidos urbanos (RSU), quer se trate de resíduos urbanos, hospitalares ou tóxicos e perigosos, é, sem dúvida aquele que é mais do conhecimento pelo público como potencial fonte de emissões de dioxinas, estando assim sujeito a grande pressão por parte deste e dos media. Possivelmente por estas razões existe, na generalidade, um melhor conhecimento dos níveis de emissão neste tipo de unidades, que são fruto de intenso estudo analítico [4]. Assim, no que diz respeito às emissões de unidades de incineração de resíduos sólidos, os níveis de incerteza são de facto menores uma vez que as condições de amostragem são mais favoráveis e as investigações efectuadas sobre estas últimas fontes resultaram num melhor conhecimento dos mecanismos de formação e decomposição destes compostos, principalmente nos sistemas de incineração de resíduos urbanos [14]. Na tabela I apresentam-se dados dos teores típicos de dioxinas e dibenzofuranos presentes em gases de exaustão de incineradoras de resíduos sólidos urbanos.

\subsection{Veículos automóveis}

Conforme se referiu anteriormente, a exaustão de gases de escape de veículos automóveis é ainda responsável pela emissão de compostos desta natureza para a atmosfera. Compostos halogenados como o dibromometano e o dicloroetano são adicionados à gasolina com chumbo para evitar a deposição de compostos de chumbo nos motores. A ausência

Tabela I - Emissões de PCDD/PCDF nos gases de exaustão de um incinerador de resíduos sólidos urbanos (RSU) de segunda geração (média de 22 amostras)

\begin{tabular}{lclc}
\hline PCDD & $\mathbf{n g} / \mathbf{N m}^{3}$ & PCDF & $\mathbf{n g} / \mathbf{N m}^{3}$ \\
\hline $2,3,7,8-\mathrm{Cl}_{4} \mathrm{DD}$ & 0,5 & $2,3,7,8-\mathrm{Cl}_{4} \mathrm{DF}$ & \\
Total das espécies $\mathrm{Cl}_{4} \mathrm{DD}$ & 11 & Total das espécies $\mathrm{Cl}_{4} \mathrm{DF}$ & 72 \\
$1,2,3,7,8-\mathrm{Cl}_{5} \mathrm{DD}$ & 2,3 & $1,2,3,7,8-\mathrm{Cl}_{5} \mathrm{DF}$ & 7,8 \\
Total das espécies $\mathrm{Cl}_{5} \mathrm{DD}$ & 24 & Total das espécies $\mathrm{Cl}_{5} \mathrm{DF}$ & 112 \\
$1,2,3,4,7,8-\mathrm{Cl}_{6} \mathrm{DD}$ & 2,9 & $1,2,3,4,7,8-\mathrm{Cl}_{6} \mathrm{DF}$ & 13,1 \\
$1,2,3,6,7,8-\mathrm{Cl}_{6} \mathrm{DD}$ & 4,0 & $1,2,3,6,7,8-\mathrm{Cl}_{6} \mathrm{DF}$ & 12,0 \\
$1,2,3,7,8,9-\mathrm{Cl}_{6} \mathrm{DD}$ & 3,6 & $1,2,3,7,8,9-\mathrm{Cl}_{6} \mathrm{DF}$ & 1,1 \\
Total das espeécies $\mathrm{Cl}_{6} \mathrm{DD}$ & 41 & Total das espécies $\mathrm{Cl}_{6} \mathrm{DF}$ & 98 \\
$1,2,3,4,6,7,8-\mathrm{Cl}_{7} \mathrm{DD}$ & 27,3 & $1,2,3,4,6,7,8-\mathrm{Cl}_{7} \mathrm{DF}$ & 71,0 \\
Total das espécies $\mathrm{Cl}_{7} \mathrm{DD}$ & 57 & $1,2,3,4,7,8,9-\mathrm{Cl}_{7}-\mathrm{DF}^{-}$ & 7,0 \\
$\mathrm{Cl}_{8} \mathrm{DD}$ & 148 & Total das espécies $\mathrm{Cl}_{7} \mathrm{DF}$ & 106 \\
Total das espécies $\mathrm{Cl}_{4}-\mathrm{Cl}_{8} \mathrm{DF}$ & 281 & Cl & 95 \\
I-TEQ & 11,8 & Total das espécies $\mathrm{Cl}_{4}-\mathrm{Cl}_{8} \mathrm{DF}$ & 483 \\
\hline
\end{tabular}

Fonte: $[24]$ 
daqueles compostos na gasolina sem chumbo faz com que a emissão de dioxinas e dibenzofuranos seja, neste caso, consideravelmente inferior $[15]$.

\subsection{Fontes diversas}

Na Tabela II apresentam-se valores estimados para as emissões anuais de dioxinas e congéneres para a atmosfera no Reino Unido, referidas a 1995, por sectores de actividade. É de assinalar neste dados a elevada contribuição da incineração de Resíduos Sólidos Urbanos, que resulta de estarem ainda em funcionamento no Reino Unido, unidades de incineração de primeira e de segunda geração. Grande parte destas unidades vai ter de instalar sistemas adicionais de purificação dos gases efluentes de forma a cumprir teores de dioxinas e dibenzofuranos inferiores a $0,1 \mathrm{ng} / \mathrm{Nm}^{3}$.

\section{TEORES DE DIOXINAS E DIBENZOFURANOS NO MEIO AMBIENTE}

As dioxinas e dibenzofuranos emitidos a partir dos processos de combustão são transportados através da atmosfera, depositando-se nos oceanos, lagos e no próprio solo. Devido à sua deposição no meio aquático e à sua baixa solubilidade em água, estes compostos vão ficando acumulados nos sedimentos. Por esta razão, o estudo analítico dos sedimentos permite avaliar quais as fontes que originaram estes contaminantes e em que altura foram emitidos. Deste modo, sabe-se que, anteriormente a 1940, apenas se produziram emissões residuais de PCDD e dibenzofuranos policlorados (PCDF), e apenas a partir desta data se começaram a verificar emissões significativas. Estes dados estão de acordo com o início das actividades

Tabela II - Estimativa das emissões anuais de dioxinas e congéneres referidos a 1995, no Reino Unido

\begin{tabular}{lc} 
Processo & $\begin{array}{c}\text { Estimativa de emissões } \\
\text { para a atmosfera (g I-TEQ/ano) }\end{array}$ \\
\hline Produção de coque & 2 \\
Combustão de carvão & $5-67$ \\
Combustão de óleos residuais & $0,8-24$ \\
Combustão de madeira & $1,4-2,9$ \\
Combustão de palha & $3,4-10$ \\
Combustão de pneus & 1,7 \\
Combustão de gás de aterros & $1,6-5,5$ \\
Unidades sinterização & $29-54$ \\
Siderurgias & $3-41$ \\
Fundições (não-ferrosos) & $5-35$ \\
Cimentos a & $0,2-11$ \\
Calcários & $0,04-2,2$ \\
Vidro & $0,005-0,01$ \\
Cerâmica & $0,02-0,06$ \\
Compostos halogenados & 0,02 \\
Pesticidas & $0,1-0,3$ \\
Combustão de RSU & $460-580$ \\
Combustão de resíduos químicos & $1,5-8,7$ \\
Combustão de resíduos hospitalares & $18-88$ \\
Combustão de lamas de ETARS & $0,7-6$ \\
Asfaltos & 1,6 \\
Crematórios & $1-35$ \\
Combustão doméstica & $22-52$ \\
Trafego automóvel & $1-45$ \\
Fogos naturais & $0,4-12$ \\
TOTAL PARA O REINO UNIDO & $560-1100$ \\
&
\end{tabular}

Fonte : $|23|$ industriais de produção de compostos clorados.

\section{IMPACTO DAS DIOXINAS E DIBENZOFURANOS SOBRE OS SERES HUMANOS}

Nos países mais industrializados do Norte da Europa (Alemanha, Reino Unido, Holanda) os níveis de dioxinas encontrados em seres humanos começam a estar perigosamente próximos da dose que se estima ser capaz de produzir os primeiros sintomas de toxicidade em seres humanos e animais [16]. Os efeitos sobre seres humanos expostos a doses baixas de dioxinas e dibenzofuranos incluem alterações do seguinte tipo : imunossupressão, com consequente diminuição das defesas do organismo contra agentes patogénicos vindos do exterior; doença hepática, com aumento de volume do fígado e acumulação intracelular de gordura (com possível evolução para cirrose hepática); possível redução da contagem de espermatozóides viáveis, o que poderia explicar parcialmente a diminuição acentuada, nos últimos 50 anos, dos níveis de fertilidade; indução enzimática dos enzimas hepáticos de destoxificação (família dos citocromos P450); alterações da coagulação sanguínea. Na Holanda foram detectados casos de derrame sanguíneo cerebral em recémnascidos, que foram associados a exposições a doses medianamente elevadas de dioxinas contidas no leite materno [16]. Estes derrames podem estar associados a uma deficiência em vitamina $\mathrm{K}$ (lipossolúvel), que pode aparecer quando há lesão das células do fígado, e uma redução consequente da quantidade de ácidos biliares incluídos na bílis. Esta redução origina uma má absorção de compostos lipossolúveis por deficiente emulsão das gorduras no duodeno, que terá afectado negativamente a absorção de vitamina $\mathrm{K}$, resultando nos derrames descritos.

O consumo de peixe por populações humanas, em zonas costeiras do Mar Báltico, foi também associa- 
do aos primeiros sintomas de exposição a dioxinas em doses medianamente elevadas [17]. As dioxinas estariam neste caso contidas na gordura do peixe, sendo este um bom exemplo da contaminação através da cadeia alimentar. A entrada destes contaminantes no corpo humano faz-se principalmente por via indirecta, através da cadeia alimentar [18], sendo menos importante a via de contacto directo com a pele e a via respiratória [19]. Os produtos animais contêm apenas um determinado número de isómeros tóxicos mais estáveis [20], e diversos estudos [21] demonstram que uma importante percentagem das dioxinas e dibenzofuranos, procedentes de sedimentos e cinzas volantes, penetram na cadeia trófica por bioacumulação em organismos aquáticos.

Uma vez o contaminante presente no corpo humano tende a acumular-se em diversas zonas com elevado teor em gordura, tais como o tecido adiposo e o leite materno. Em 1989 efectuaram-se diversas análises de leite humano, em que foi detectada a presença de PCDD e PCDF [22]. Os níveis encontrados indicam que não há diferenças entre zonas industrializadas e outras. Mais de $50 \%$ das dioxinas encontradas são do tipo octaclorodibenzo-p-dioxina, em gamas de concentração entre 200 e 2000 pg/g de gordura. Uma conclusão importante destes estudos é a de que a concentração de PCDD e PCDF no leite materno decresce à medida que aumenta o número de filhos que tenham sido amamentados pelas suas mães, o que indica que a amamentação é uma via importante de transferência das dioxinas da mãe para o filho. Além disso, tem-se verificado, por meio de estudos cinéticos, que os isómeros mais lipofílicos são os que se eliminam mais rapidamente através do leite materno. Vartiainen e Liimatainen [16] demonstraram que as dioxinas e dibenzofuranos são cancerígenos no Homem (cerca de 20 anos após a exposição), como aliás já tinha sido demonstrado em animais, embora os mecanismos concretos da geração de tumores não estejam ainda completa- mente esclarecidos. Os "efeitos letais" da exposição teriam assim um período de incubação de cerca de 20 anos, o que explica a dificuldade, encontrada até 1991, em estabelecer um nexo de causalidade entre exposição a dioxinas - tanto no caso de acidentes graves (exposição a doses altas em curto período de tempo) como nos casos de trabalhadores expostos a doses mais baixas por períodos consideráveis - e o aparecimento de cancro no Homem.

\section{CONCLUSÃO}

Do exposto, conclui-se que os compostos policlorados da família das dioxinas e os dibenzofuranos constituem um problema de que só se tem vindo a tomar consciência recentemente, mas que tudo indica ser de enorme gravidade, principalmente devido á facilidade de bio-acumulação, persistência e toxicidade. Além disso, o seu efeito não se restringe a áreas industriais limitadas uma vez que as fontes que os originam são muito variadas e estes contaminantes são susceptíveis de serem transportadas pelo vento através da atmosfera até zonas razoavelmente afastadas da sua fonte de geração.

\section{REFERÊNCIAS}

1. Gilman, H.; Dietrich, J.I., I. Amer. Chem Soc. $79,1439(1957)$

2. Sanderman, W.; Stockman, H.; Casten, R., Chem. Ber., 90, 690 (1957).

3. Kimmig, 1.; Schultz, K.H., Dermatologia, 115,540 (1957).

4. Rappe, C., in Dioxin, Toxicological and Chemical Aspects, SP Medical and Scientific Books, London, 1978.

5. Fanelli, R., Garttini, S., "Human exposure to dioxin", New Horizons in Biological Dosimetry, 167. Wiley-Liss Eds., New York, 1991.

6. Travis, C.C.; Frey, H.A.H., Science of Total Environment, 104, 97 (1991).

7. Pohjanvirta, R.; Vartiainen, T.; Uusirauva, A.; Monkkonen, J.; Tuomisto, J.; Pharmacology and Toxicology, 66, 93 (1990).

8. Vartiainen, T.; Liimatainen, A.; Kauranen, P.; Science of Total Environment, 62, 75 (1987).
9. Kringstad, K.P.; Lindstrom, K., Environmental Science Technology, 18, 236A (1984).

10. Rosenberg, C.; Kontsas, H.; Jappinen, P.; Tornaeus, I.; Hesso, A, Vainio, H., Chemosphere, $29(9-11), 1971$ (1994).

11. Halonen, I.; Tarhanen, J.; Oksanen, J.; Vilokki, H.; Vartiainen, T.; Ruuskanen, J., Chemosphere, 16, 1759, 1993.

12. Someshwar, A.V.; Jain, A.K.; Whittemore, R.C.; Lafleur, L.E.; Gillespie, W.J., Chemosphere, 20(10-12), 1715, 1990

13. Swedish Environmental Protection Agency, Report 4008, Stockholm, 1992.

14. Penner, S.S.; Li, C.P.; Richards, M.B.; Wiesenhahn, D.F., Science of Total Environment, 104 35 (1991).

15. Muller, M.D.; Buser, H.R., Environmental Science Technology, 20, 1151 (1986).

16. Vartiainen, T.; Liimatainen,A., Mutation Res., 169, 29 (1986).

17. Kronberg, L.; Vartiainen, T., Mutation Res. 206, 177 (1988).

18. McLachlan, M.; Thoma, H.; Reissinger, M.; Hutzinger, O., Dioxin 89, Toronto, 1989.

19. Keenan, R.E.; Sauer, M-M.; Lawrence, F.H. Chemosphere, 19, 877 (1989).

20. Beck, H.; Eckart, K.; Mathar, W.; Wittkowski. R., Chemosphere, 19, 655 (1989).

21. Kuel, D.W.; Cook, P.M.; Batterman, A.R.; Lothenback, D.B.; Butterworth, B.C.; Johnson, D.L., Chemosphere, 14, 427 (1985).

22. Birmingham, B.; Thorpe, B.; Frank, R.; Clement, R.; Tosine, H.; Fleming, G., Chemosphere, 19, 507 (1989).

23. Her Majesty's Inspectorate of Pollution, "A Review of Dioxin Emissions in the UK", DoE Report $\mathrm{N}^{2}$ DoE/HMIP/RR/95/004, Sector $\mathrm{N}^{0} 2.1$, DoE, London, 1995.

24. Fielder, H.; Hutzinger, O., Toxicological and Environmental Chemistry, 29, 157 (1991).

t as concentraçôes de poluentes em gases indicam-se expressas em unidades de massa por $\mathrm{m}^{3} \mathrm{em}$ condiçôes PTN $\left(\mathrm{Nm}^{3}\right)$

(*) Departamento de Engenharia Química, Instituto Superior Técnico, Av. Rovisco Pais, 1096 Lisboa Codex

(**) Faculdade de Farmácia; Universidade de Lisboa, Av. das Forças Armadas, 1600 Lisboa

${ }^{* * *}$ ) Centro de Tecnologias Ambientais, Divisāo de Ambiente, Energia e Segurança, Instituto de Soldadura e Qualidade, Apartado 119. 2781 Oeiras Codex 